\section{Tissue engineered constructs for the replacement of laboratory animals: Biomimetics and microfluidics}

\author{
Maria Grazia Cascone, Luigi Lazzeri, \\ Elisabetta Rosellini \\ Department of Civil and Industrial \\ Engineering, University of Pisa, Pisa, \\ Italy
}

\begin{abstract}
The purpose of this contribution is to highlight how Biomimetics and Microfluidics can be used as tools for the in vitro creation of $3 \mathrm{D}$ systems to be employed as a valid alternative to animal models. Microfluidic bioreactors can be used for cell culture on biomimetic scaffolds as in vitro tissue and organ models with the aim to develop alternative methods to in vivo experiments. Several types of biomimetic scaffolds have been prepared using different techniques of production and functionalization. A microfluidic bioreactor was designed for perfusion culture of cardiomiocytes seeded onto biomimetic alginate/gelatin sponges and high viability of the resulting cardiac constructs, under dynamic flow culture, was observed.
\end{abstract}

\section{Introduction}

The purpose of this work is to highlight how Biomimetics and Microfluidics can be used as tools for the in vitro creation of $3 \mathrm{D}$ systems to be employed as a valid alternative to animal models.

The use of animals for biomedical research purposes has been the subject of debate for many years. Identification of the importance of welfare for animals used in science has pushed towards the development of methods to replace animals in research. Animal models often show limits in the reproduction of specific human conditions. Human pathologies can be induced in animal models but the molecular mechanisms that guide their onset and progression are often significantly different. The proven inefficacy in humans of some drugs, successfully tested on animals, are symptoms of the animal model's inability to reproduce effectively human physiology. Over the last ten years the enormous potential of $3 \mathrm{D}$ in vitro systems has been increasingly evident as they allow the cells to growth and interact each other and with the extracellular matrix (ECM) in all spatial dimensions, mimicking accurately the microenvironment in which cells thrive in vivo.

Most of the focus is on 3D scaffoldbased systems in which the 3D structure is achieved through a 3D support matrix called "scaffold".

The scaffold should not only function as a support for cell adhesion, but it should also be "bioactive" ie it should recapitulate the natural environment and provide various cues to direct cell processes and differentiation as well as the ECM does in vivo.

In order to be able to create in vitro models capable of replacing animal models and therefore able to perfectly mimic the microenvironment in vivo, a valuable aid could also come from microfluidics. The microfluidic technology also called Lab on-a-chip, can provide micro-scale complex structures and well-controlled parameters to mimic the in vivo environment of cells. Microfluidics can supply useful model systems to investigate complex phenomena under combination of multiple controllable biochemical and biophysical microenvironments.

In light of the above, it seems logical to think that Biomimetics and Microfluidics can be combined to develop alternative methods to in vivo experiments through the culture of cells on biomimetic scaffolds inside microfluidic bioreactors.

\section{Results}

Aim of the research activity carried out by our group in the last years years has been the production and characterization of materials to be used as biomimetic scaffolds for the in vitro creation of 3D systems. Several procedures have been used to create biomimetic systems capable of influencing cell behavior, as ECM does in vivo.

Biomimetic scaffolds have been produced by blending of different natural polymers. In particular scaffolds based on blends of proteins and polysaccharides have been designed as surrogates of the native ECM. A scaffolding system where a protein component blends with a polysaccharide component, mimicking composition and interactions in the native ECM, would be a logical design to guide the division, growth, and development of residing cells.

3D porous sponges based on mixtures of alginate with collagen or gelatin at 20:80 weight ratios, were produced by freeze-drying and subsequent ionic and chemical crosslinking procedures, for application in cardiac tissue regeneration. ${ }^{1}$

Bioactive polymeric systems have been produced using traditional and innovative functionalization techniques such as: 1)
Correspondence: Maria Grazia Cascone, Department of Civil and Industrial Engineering, University of Pisa, Largo Lucio Lazzarino 2, Pisa, Italy.

E-mail: maria.grazia.cascone@unipi.it

Key words: Scaffold; functionalization; alternative methods.

Conference presentation: this paper was presented at the Second Centro 3R Annual Meeting - 3Rs in Italian Universities, 2019, June 20-21, University of Genoa, Italy.

Received for publication: 28 October 2019.

Accepted for publication: 6 November 2019.

This work is licensed under a Creative Commons Attribution NonCommercial 4.0 License (CC BY-NC 4.0).

CCopyright: the Author(s), 2019

Licensee PAGEPress, Italy

Biomedical Science and Engineering 2019; 3(s2):83 doi:10.4081/bse.2019.83

Covalent bonding of peptide sequences like: Arg-Gly-Asp (RGD), Tyr-Ile-Gly-SerArg (YIGSR), Arg-Glu-Asp-Val (REDV), etc; 2) Direct loading with active agents; 3) Loading with micro-/nano-particles containing active agents; 4) Molecular Imprinting. ${ }^{2}$

Biomimetic scaffolds were obtained by surface chemical immobilization of bioactive peptide sequences on synthetic bioresorbable polymers, treated by alkaline hydrolysis, through the formation of amide bonds. ${ }^{3}$

Biomimetic non-woven meshes based on poly(lactic acid) (PLLA) hollow microfibers obtained by dry-wet spinning were prepared by loading the fibers with poly(lactic-co-glycolic acid) (PLGA) microparticles containing active agents. ${ }^{4}$

Biomimetic poly(vinyl alcohol) hydrogels, obtained by freezing-thawing cycles, and biomimetic gelatin/PLLA and agarose/PLLA sponges, produced by emulsion freeze-drying, were prepared by loading with PLGA microparticles, containing active agents.

Innovative biomimetic scaffolds were also produced by functionalization through molecularly imprinted polymers. Molecular imprinting technology permits the production of synthetic polymers capable of selectively linking themselves to a specific substance, called template. Bioactive scaffolds were obtained by deposition on their surface of molecularly imprinted particles with recognition properties towards bioactive molecules able to promote desired cellular responses. ${ }^{5}$

Recently our research activity has 


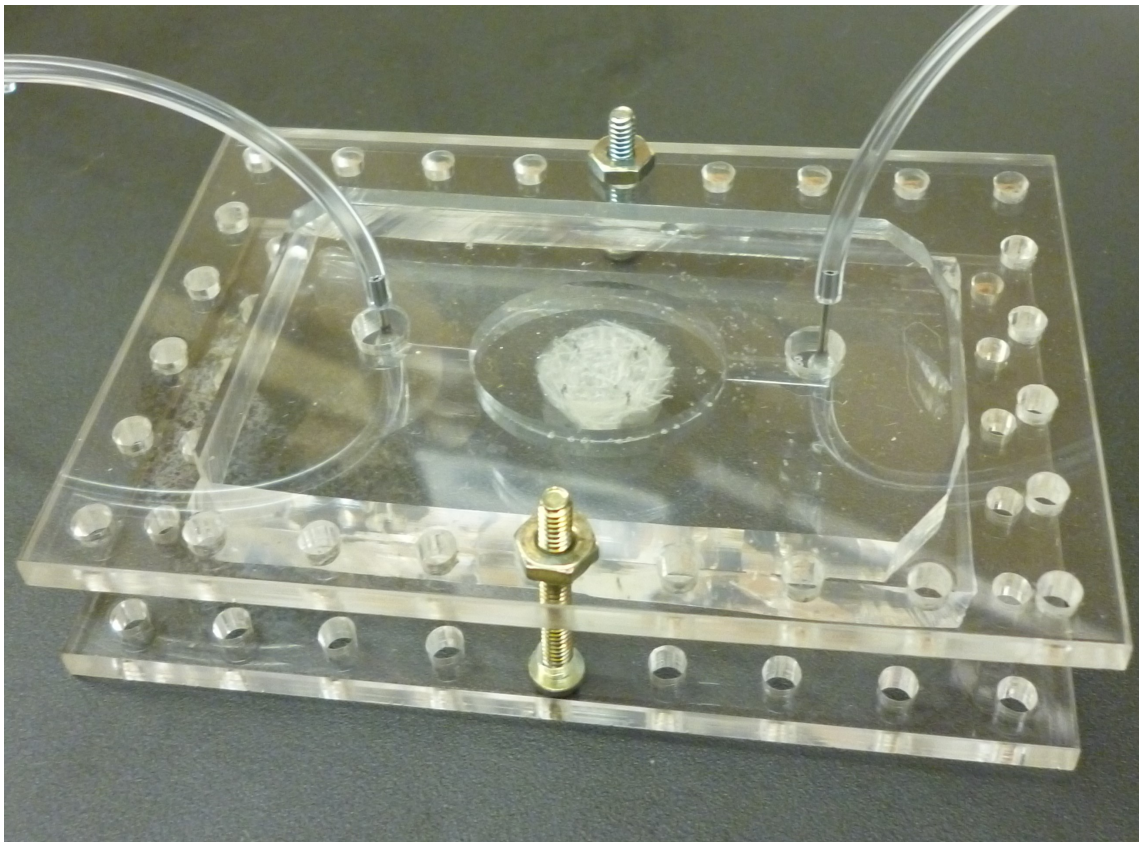

Figure 1. Microfluidic bioreactor.

focused on microfluidics. As an example, a microfluidic bioreactor was designed for perfusion culture of cardiomiocytes onto biomimetic alginate/gelatin sponges (Figure 1) and high viability of the resulting cardiac constructs, under dynamic flow culture, was observed. 5
3D environments. The combination of microfluidic bioreactors with 3D biomimetic scaffolds seems to offers great potential for in vivo-like applications such as alternatives to animal experimentation.

\section{References}

1. Rosellini E, Zhang YS, Migliori B, et al. Protein/polysaccharide-based scaffolds mimicking native extracellular matrix for cardiac tissue engineering applications. J Biomed Mater Res A 2018;106:769-81.

2. Tallawi M, Rosellini E, Barbani N, et al. Strategies for the chemical and biological functionalization of scaffolds for cardiac tissue engineering: A review. J R Soc Interface 2015;12:20150254.

3. Rosellini E, Cristallini C, Guerra G, Barbani N. Surface chemical immobilization of bioactive peptides on synthetic polymers for cardiac tissue engineering. J Biomater Sci Polym Ed 2015;26:51533.

\section{Conclusions}

Biomimetic scaffolds are systems characterized by chemical-physical signals and/or by a structure that mimics the ECM, allowing to control and influence the specific cellular response. Microfluidic devices can readily create complex dynamic microscale environments to mimic in vivo
4. Lazzeri L, Cascone MG, Quiriconi S, et al. Biodegradable hollow microfibres to produce bioactive scaffolds. Polym Int 2005;54:101-7.

5. Rosellini E, Barbani N, Giusti P, et al. Novel bioactive scaffolds with fibronectin recognition nanosites based on molecular imprinting technology. J Appl Pol Sci 2010;118:3236-44. 